\title{
Open-Source Conceptual Sizing Models for the Hyperloop Passenger Pod
}

\author{
Jeffrey C. Chin, Justin S. Gray*, Scott M. Jones*, Jeffrey J. Berton* \\ NASA Glenn Research Center, Cleveland, $\mathrm{OH}$
}

\begin{abstract}
Hyperloop is a new mode of transportation proposed as an alternative to California's high speed rail project, with the intended benefits of higher performance at lower overall costs. It consists of a passenger pod traveling through a tube under a light vacuum and suspended on air bearings. The pod travels up to transonic speeds resulting in a 35 minute travel time between the intended route from Los Angeles and San Francisco. Of the two variants outlined, the smaller system includes a 1.1 meter tall passenger capsule traveling through a 2.2 meter tube at 700 miles per hour. The passenger pod features water-based heat exchangers as well as an on-board compression system that reduces the aerodynamic drag as it moves through the tube. Although the original proposal looks very promising, it assumes that tube and pod dimensions are independently sizable without fully acknowledging the constraints of the compressor system on the pod geometry. This work focuses on the aerodynamic and thermodynamic interactions between the two largest systems; the tube and the pod. Using open-source toolsets, a new sizing method is developed based on one-dimensional thermodynamic relationships that accounts for the strong interactions between these sub-systems. These additional considerations require a tube nearly twice the size originally considered and limit the maximum pod travel speed to about 620 miles per hour. Although the results indicate that Hyperloop will need to be larger and slightly slower than originally intended, the estimated travel time only increases by approximately five minutes, so the overall performance is not dramatically affected. In addition, the proposed on-board heat exchanger is not an ideal solution to achieve reasonable equilibrium air temperatures within the tube. Removal of this subsystem represents a potential reduction in weight, energy requirements and complexity of the pod. In light of these finding, the core concept still remains a compelling possibility, although additional engineering and economic analyses are markedly necessary before a more complete design can be developed.
\end{abstract}

\section{Nomenclature}

$\begin{array}{llll}A & \text { Area }\left(m^{2}\right) & L & \text { Length }(\mathrm{m}) \\ B F & \text { Blockage Factor, }\left(\frac{A_{\text {diffused }}}{A_{\text {pod }}}\right) & M & \text { Mach Number } \\ C_{p} & \text { Heat Capacity, Constant Pressure }\left(\frac{J}{k g-K}\right) & \dot{m} & \text { Mass Flow Rate }\left(\frac{k g}{s}\right) \\ c_{\text {solar }} & \text { Solar Irradiance Gross Adjustment } & n & \text { Number of Pods } \\ D & \text { Diameter }(m) & N u & \text { Nusselt Number }\left(\frac{h D}{k}\right) \\ g & \text { Acceleration of Gravity }\left(\frac{m}{s^{2}}\right) & P & \text { Pressure }\left(\frac{N}{m^{2}}\right) \\ G & \text { Solar Irradiance }\left(\frac{W}{m^{3}}\right) & P r & \text { Prandtl Number }\left(\frac{v}{\alpha}\right) \\ G r & \text { Grashof Number }\left(\frac{g \beta \Delta T D^{3}}{v^{2}}\right) & Q & \text { Heat Flow Rate }(\mathrm{W}) \\ h & \text { Heat Transfer Coefficient }\left(\frac{W}{m^{2}-K}\right) & r & \text { Radius }(\mathrm{m}) \\ k & \text { Thermal Conductivity }\left(\frac{W}{m-K}\right) & R a & \text { Rayleigh Number }(G r \cdot P r) \\ & & T & \text { Temperature }(\mathrm{K})\end{array}$

*Aerospace Engineer, Propulsion Systems Analysis Branch, Mail Stop 5-10, AIAA Member 


$\begin{array}{ll}\alpha & \text { Thermal Diffusivity }\left(\frac{m^{2}}{s}\right) \\ \beta & \text { Volume Coefficient of Expansion }\left(\frac{1}{K}\right) \\ \gamma & \text { Heat Capacity Ratio } \\ \epsilon & \text { Total, Hemispherical Emissivity } \\ \eta_{c} & \text { Adiabatic Compressor Efficiency }\end{array}$

$\pi_{c} \quad$ Compressor Pressure Ratio

$\rho_{r} \quad$ Total, Hemispherical Reflectivity

$\sigma \quad$ Stefan-Boltzmann Constant $\left(\frac{W}{m^{2} \cdot K^{4}}\right)$

$v \quad$ Kinematic Viscosity $\left(\frac{m^{2}}{s}\right)$

\section{Introduction}

Hyperloop is a conceptual transportation system designed to lower costs and travel times relative to California's current high-speed rail project. ${ }^{1}$ Elon Musk and a team of engineers from Tesla Motors and the Space Exploration Technologies Corporation (SpaceX) proposed the idea in August 2013 as an open design to be vetted and further refined through public contribution. The concept deviates from existing high-speed rail designs by eliminating the rails, enclosing the passenger pod in a tube under a partial vacuum, and suspending the pod on air bearings. Propulsion is handled by a set of linear electromagnetic accelerators mounted to the tube with the entire system held above ground on concrete columns maintaining a relatively straight trajectory.

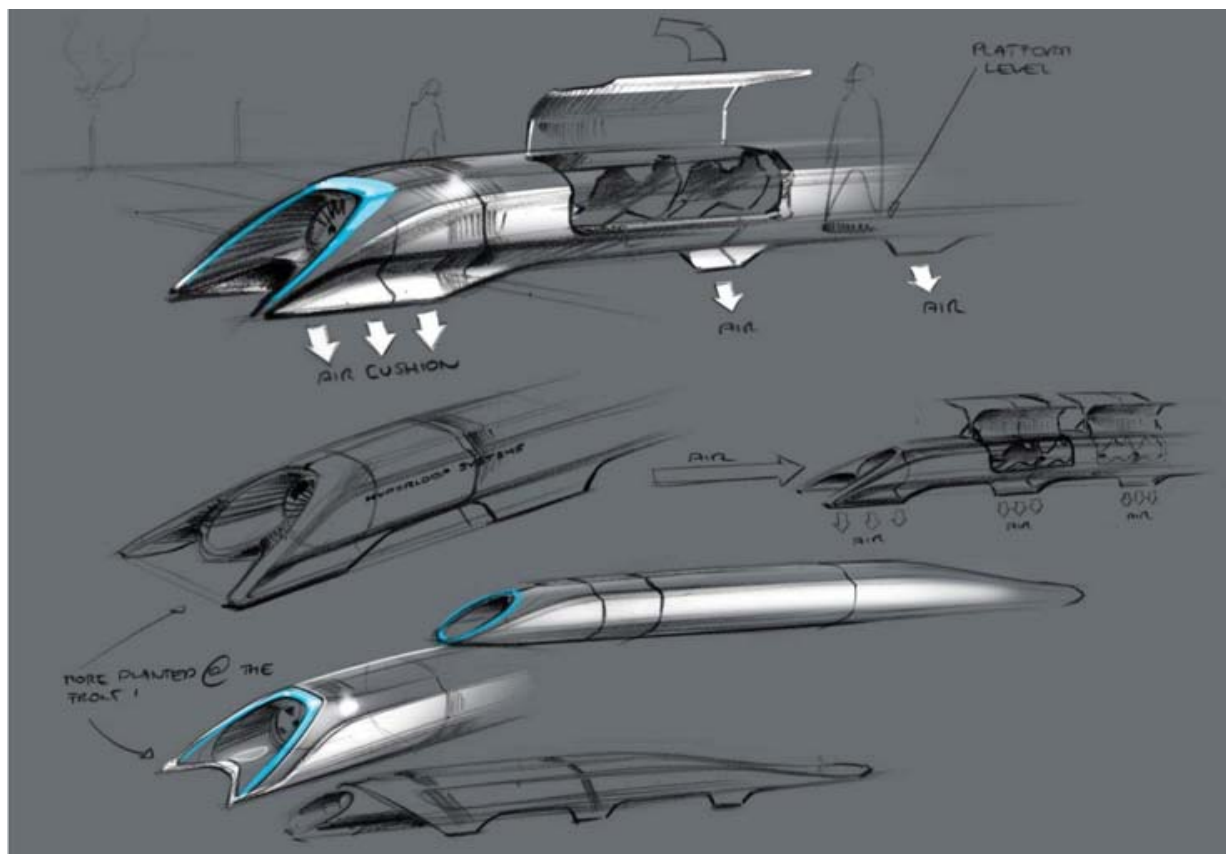

Figure 1: Hyperloop-alpha concept sketch of the passenger pod. ${ }^{1}$

Although Hyperloop is similar to other vacuum tube train (VacTrain) concepts, ${ }^{2}$ the soft vacuum represents a distinct difference. It allows the pod to run on air-bearings, thus removing the need for a magnetic levitation system used on other VacTrain designs. The air bearings require a source of pressurized air, which is provided by a compressor powered by on-board batteries. Since Hyperloop operates at transonic speeds and a low pressure environment, the design of the pod compression system can be likened to the compressor design for aircraft turbomachinery. Furthermore, the aerodynamic concerns arising from constricted flow through a tube are prevalent in the design of inlets and nozzles on aircraft engines and the entire system faces similar weight and volume constraints. For these reasons, the modeling approach applied here is inspired heavily by methods for aircraft sizing and turbine engine cycle analysis.

Musk's original Hyperloop proposal includes individual high-level analyses of many major subsystems such as the pod compression system, elevated support structure, and propulsion system. While this demonstrates the basic viability of the concept, it does not address significant interdisciplinary couplings inherent in the Hyperloop system. These couplings introduce certain constraints that limit the degrees of freedom available in the design space. The major contribution of this work is to identify the key couplings that constrain the system and adapt aircraft sizing methods to construct a conceptual design process that accounts for them. The most significant interdisciplinary coupling arises between the passenger pod size and travel tube size. Aerodynamic concerns make it impossible to vary both pod size and tube size independently of 
each other. An additional less severe coupling also exists between the compression system and the thermal management system. The interdisciplinary coupling demands the use of an iterative sizing procedure that balances the various systems to provide a feasible design for any given set of design variables. The results of this sizing process show that Hyperloop remains plausible, but certain estimates from the original proposal may have been overly optimistic due to each discipline being approached independently.

The sizing process is a necessary precursor before ultimately performing a design optimization of Hyperloop with the overall objectives such as reducing construction costs, operational costs, and travel time. Performing this broader optimization is outside the scope of this work and is reserved for future investigation. The fully integrated system model is constructed using OpenMDAO, a Python-based framework for the design and optimization of highly coupled systems. ${ }^{3}$ This work focuses on the aerodynamics and thermodynamics of the passenger pod itself. As such, it ignores many additional key sub-systems that will also have a large impact on the final design. A more realistic design optimization will require more detailed models of the investigated systems and additional analyses for economic and structural models. Adding all of this will result in significant growth in complexity of the Hyperloop model. OpenMDAO can provide the means to manage growing model complexity in an efficient and flexible manner. Musk's original work was released with the stated goal of jump starting a crowd sourced design effort. In that spirit, all of the analyses used in this work are released under the Apache V2.0 open source license so that they can potentially serve as a foundation for future work. Links to the source code can be found in appendix A

This paper addresses three overarching system challenges related to the passenger pod sizing. Section II describes the overall connectivity of the Hyperloop subsystems. Section III clarifies a more stringent coupling between the passenger pod and tube size and the impact this has on the passenger pod dimensions and performance. Section IV investigates the compressor and battery requirements for the proposed Los Angeles to San Francisco route, considering the effects of maximum travel speed. Lastly, section V investigates the thermal equilibrium of the travel tube to provide a means of sizing the thermal management system.

\section{Hyperloop Model Overview}

The Hyperloop passenger pod was decomposed into five analyses that were connected to form the conceptual sizing model.

1. Compression System: Performance and power consumption of the compressors.

2. Mission Analysis: Estimate of travel time and velocity profile.

3. Pod Geometry: Physical dimensions of the passenger pod.

4. Tube Flow Limitations: Pod speed limitations based on choked flow restrictions.

5. Tube Wall Temperature: Equilibrium temperature of the tube.

The relationships between the systems are illustrated in Fig. 2. Feed-forward connections, or variables affecting successive subsystems, are visualized as the blue arrows in the upper-right side of the diagram. For example, the Compressor Cycle passes data to the Mission Analysis, Pod Geometry, and Tube Temperature analyses. In the lower-left side of the diagram, red arrows represent feedback connections, which establish cyclical coupling between different analyses. Table 1 summarizes the baseline values from the original proposal, which are used as design variables in this analysis. Notably, both pod radius and travel tube radius are omitted, since these variables cannot be freely varied. Instead, the assumption of a fixed area for a passenger compartment was made which, combined with the couplings shown in Fig. 2, removed these variables as design inputs. This issue is discussed in much greater detail in Section III.

The compression system and pod geometry analyses are each further subdivided into a number of subsystems. For this work, the pod geometry subsystem is primarily responsible for computing physical dimensions using high-level geometric relationships. Figure 3 shows the pod geometry breakdown into five further nested subsystems. This subdivision enhances modularity so that future work can replace these simple analyses with more advanced models (e.g. structural analyses and weight estimation for the various subsystems).

The compressor cycle performs calculations related to the thermodynamic processes of compressing, cooling, and exhausting air. The modeling approach for the compressor cycle is heavily influenced by techniques developed for the Numerical Propulsion System Simulation (NPSS) framework. NPSS was created 
Table 1: Hyperloop passenger pod design variables

\begin{tabular}{lc}
\hline \hline Design Variable & Value \\
\hline \hline Max. Pod Mach & 0.9 \\
Pod Bypass Mach & 0.95 \\
Compressor 1 Inlet Mach & 0.6 \\
Compressor 1 Pressure Ratio & 12.47 \\
Tube Static Pressure (Pa) & 99 \\
\hline
\end{tabular}

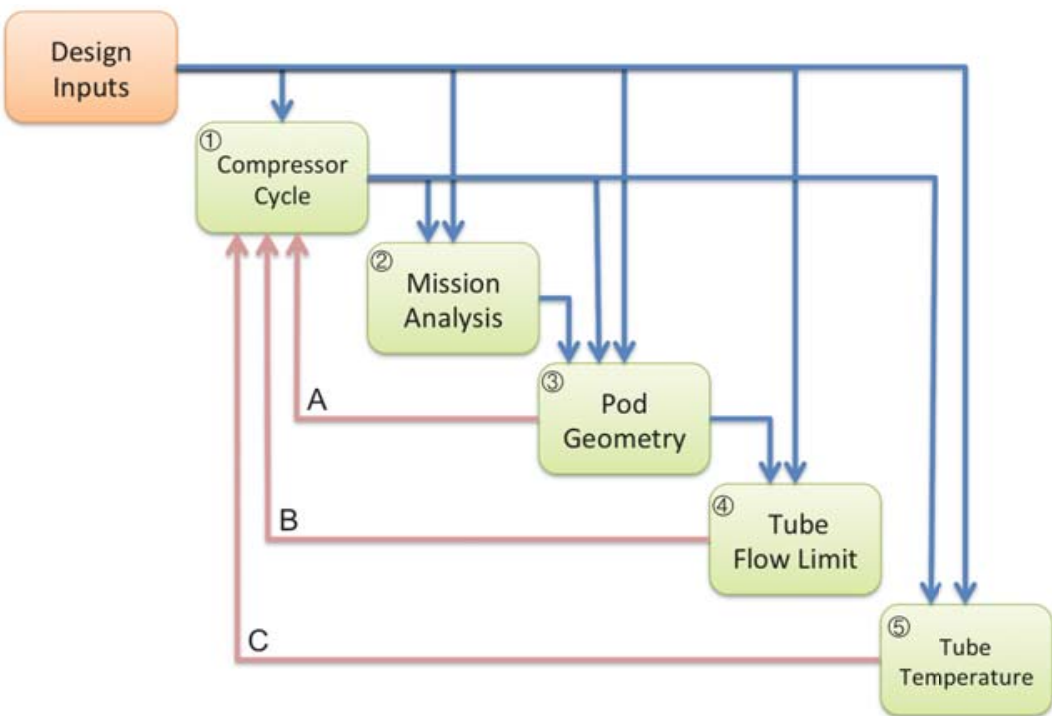

Figure 2: Workflow and dependencies between the top-level Hyperloop assemblies.

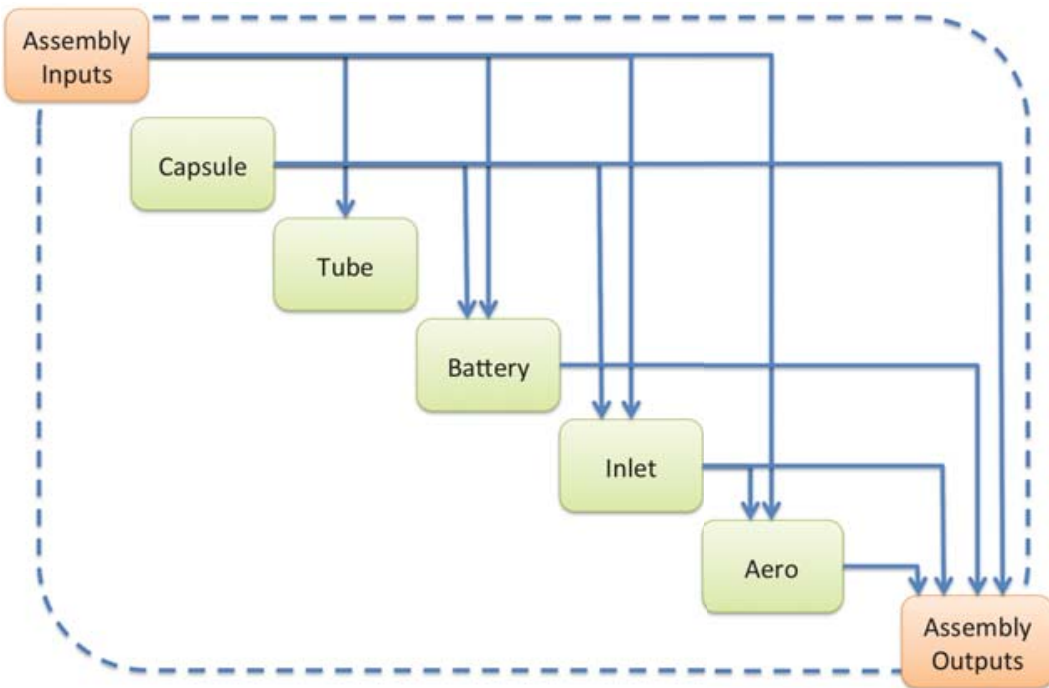

Figure 3: Expanded pod geometry assembly 
as a joint effort between United States engine manufacturers and NASA Glenn Research Center for the purpose of simulating and analyzing turbomachinery cycles for aircraft applications. ${ }^{4}$ NPSS employs a highly modular model construction where cycles get broken down into individual thermodynamic processes, which are then subsequently connected together. This object-oriented approach was followed for an open-source adaption of the tool used to predict the fluid properties and power consumption of each component in the cycle. As outlined in the original Hyperloop proposal, the compression system is modeled as two separate compressors. The first compresses the air to bypass the internal structure and passenger compartment and the second provides an additional pressure rise to reach the necessary air bearing pressure of $11 \mathrm{kPa}$. Subsequently, the required pressure ratio for the second stage is a function of the maximum pod Mach number and the first stage compressor pressure ratio. In Fig. 4 this relationship manifests as a feedback connection between the overall performance and second compressor subsystems.

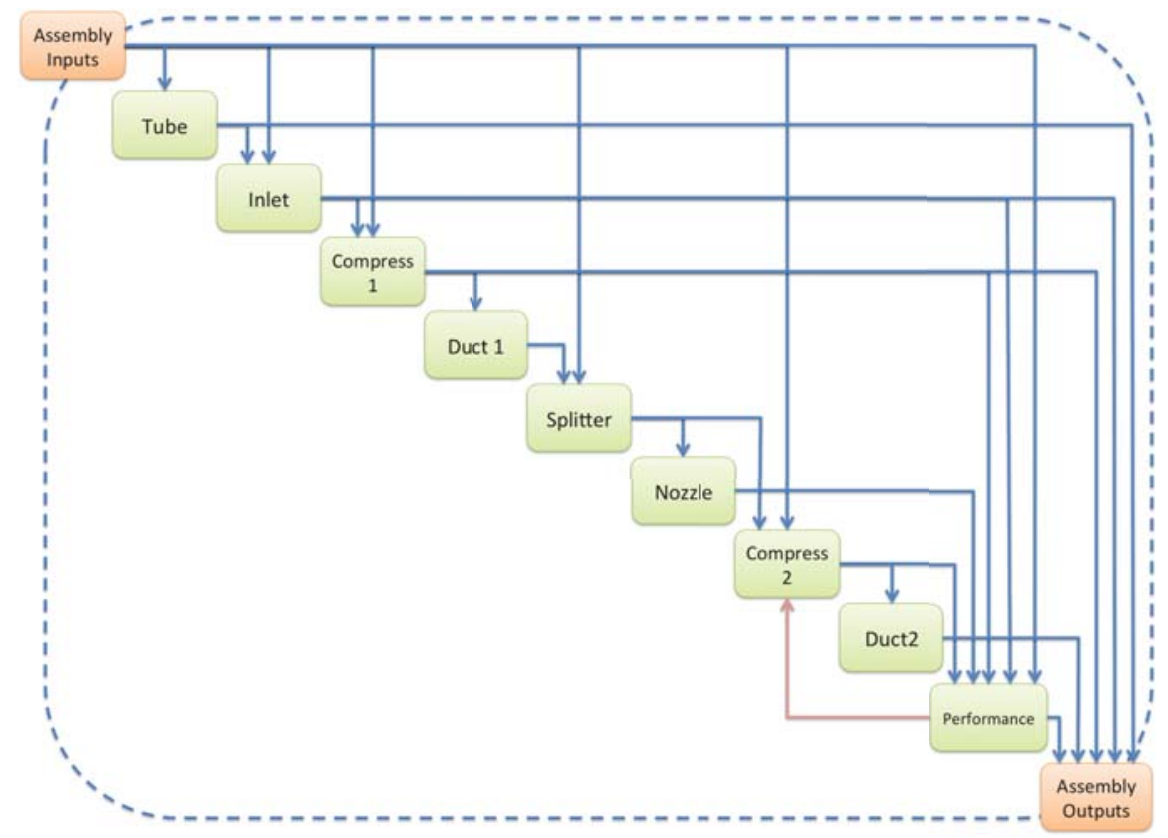

Figure 4: Expanded compressor cycle assembly

\section{Pod and Tube Sizing}

In the original Hyperloop proposal, the size of the passenger pod and the travel tube are treated as independent design variables, free to vary within reasonable ranges of each other. Two different configurations are proposed: a smaller pod for passengers and a larger pod for both passengers and cargo. The two configurations had slightly different ratios of tube area to pod area with overall dimensions summarized in Table 2 .

Table 2: Dimensions of the passenger and cargo Hyperloop designs from the original proposal.

\begin{tabular}{lcc}
\hline & Passenger & Passenger + Cargo \\
\hline$r_{\text {tube }}, m$ & 1.11 & 1.65 \\
$A_{\text {tube }}, m^{2}$ & 3.87 & 8.55 \\
$A_{\text {pod }}, m^{2}$ & 1.4 & 4.0 \\
$A_{\text {bypass }}, m^{2}$ & 2.47 & 4.55 \\
$\frac{A_{\text {bypass }}}{A_{\text {tube }}}$ & 0.64 & 0.53 \\
Max Mach & 0.95 & 0.95 \\
\hline
\end{tabular}

Smaller tube sizes are preferable because they provide lower construction costs. However, the tube size is coupled to the speed of the air as it passes around a pod of a given size. To understand this coupling, consider the following simplified situation: assume that the passenger pod is sealed with a fairing instead of an inlet. Figure 5 depicts this situation with the pod traveling through a frictionless tube from right to left, 


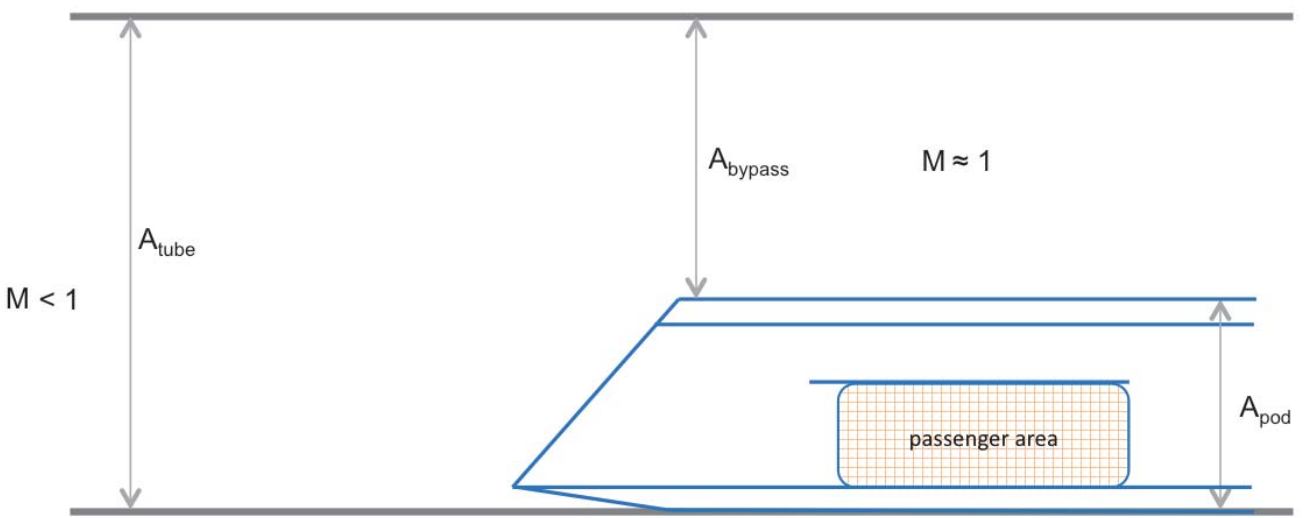

Figure 5: Longitudinal view of a passenger pod with no inlet and no compression system.

at some Mach number less than 1, through a stationary mass of air. For the purposes of analysis it is more convenient to consider the air speed relative to the pod, where the pod is stationary and the air is moving from left to right with some relative Mach number, $M_{\text {pod }}$. As the air moves around the pod, it must fit into the smaller space available to it, $A_{\text {bypass. }}$ This causes the air to accelerate to some higher Mach number, $M_{\text {bypass. }}$. From isentropic flow equations, there is a relationship between $M_{\text {pod }}$ and $M_{\text {bypass }}$ that defines an area ratio, $\frac{A_{\text {bypass }}}{A_{\text {tube }}}$, where $\gamma$ is the heat capacity ratio.

$$
\frac{A_{\text {bypass }}}{A_{\text {tube }}}=\frac{M_{\text {pod }}}{M_{\text {bypass }}}\left(\frac{1+\frac{\gamma-1}{2} M_{\text {bypass }}^{2}}{1+\frac{\gamma-1}{2} M_{\text {pod }}^{2}}\right)^{\frac{\gamma+1}{2(1-\gamma)}}
$$

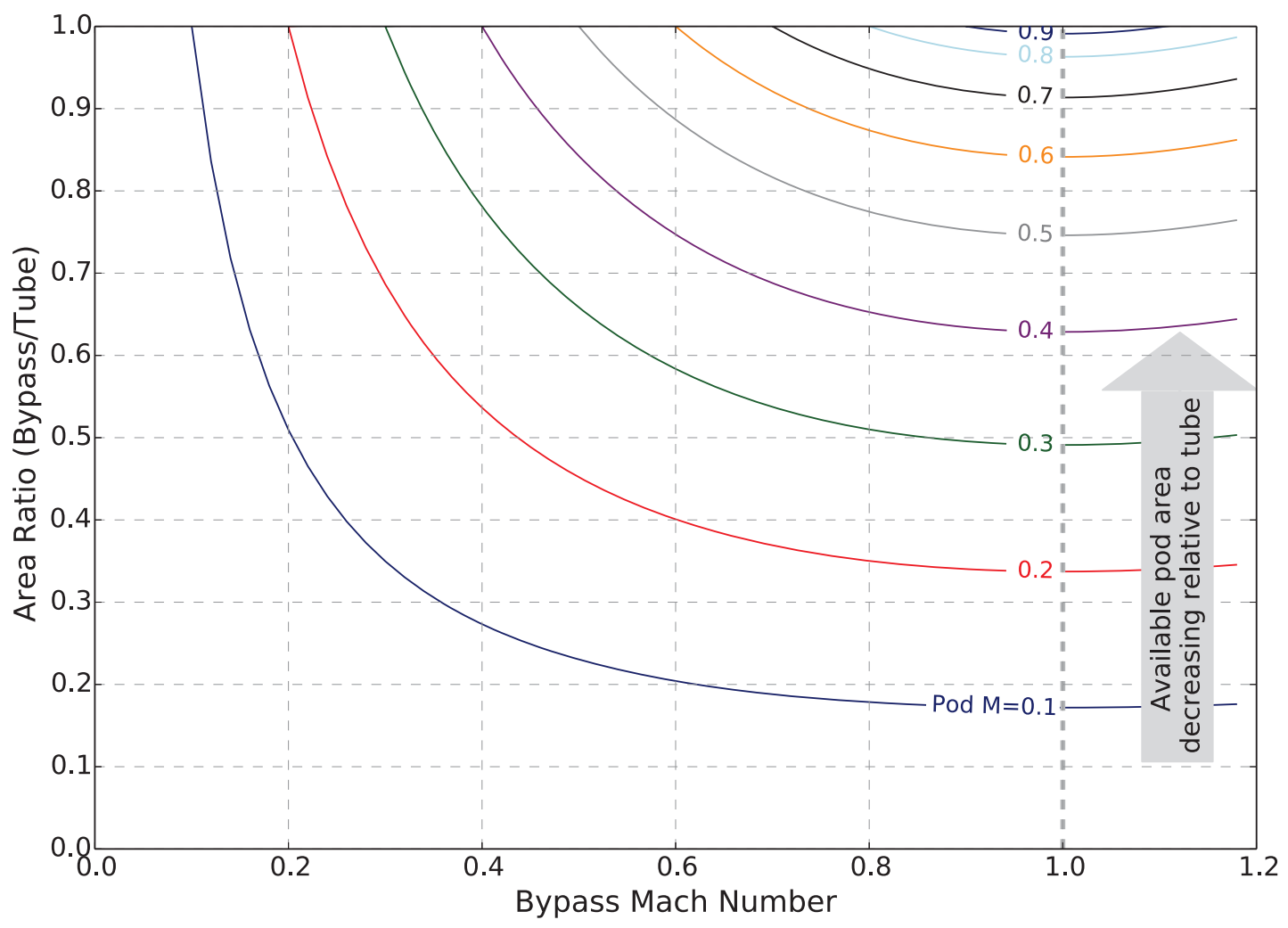

Figure 6: The required area ratio reaches a minimum value at $M_{\text {bypass }}=1$ for all values of $M_{\text {pod }}$, given the closed pod configuration illustrated in Figure 5.

Figure 6 show the effect of Eq. (1) over a range of values for $M_{\text {pod }}$ and $M_{\text {bypass }}$. For all values of $M_{\text {pod }}$, 
shown as a family of curves, the minimum required $\frac{A_{\text {bypass }}}{A_{\text {tube }}}$ occurs exactly at $M_{\text {bypass }}=1$. In other words, $M_{\text {bypass }}=1$ will always give the smallest possible $A_{\text {bypass }}$ (and hence $A_{\text {tube }}$ ) for a given $M_{\text {pod }}$ and $A_{\text {pod }}$. Thus $A_{\text {tube }}$ cannot be treated as an independent design variable without over-constraining the problem. If $A_{\text {tube }}$ is forced to be smaller than this minimum value, not all the relative mass flow can pass freely around the pod. This excess flow would accumulate to the left of the pod in Fig. 5, with the pod acting like a piston: pressurizing the air in front of it.

Furthermore, Fig. 6 also shows that $\frac{A_{\text {bypass }}}{A_{\text {tube }}}$ increases with increasing $M_{\text {pod }}$. So the faster the pod travels, the larger $A_{\text {bypass }}$ must become relative to the tube area, leaving less area for the pod. For $M_{\text {pod }}=1, \frac{A_{b y p a s s}}{A_{\text {tube }}}$ must equal 1 as well, forcing $A_{\text {pod }}$ to be 0 . Note that the values from Table 2 translate to area ratios around 0.6, which yields a much lower maximum pod speed of about Mach 0.3-0.4 for a pod without an inlet and compression system.

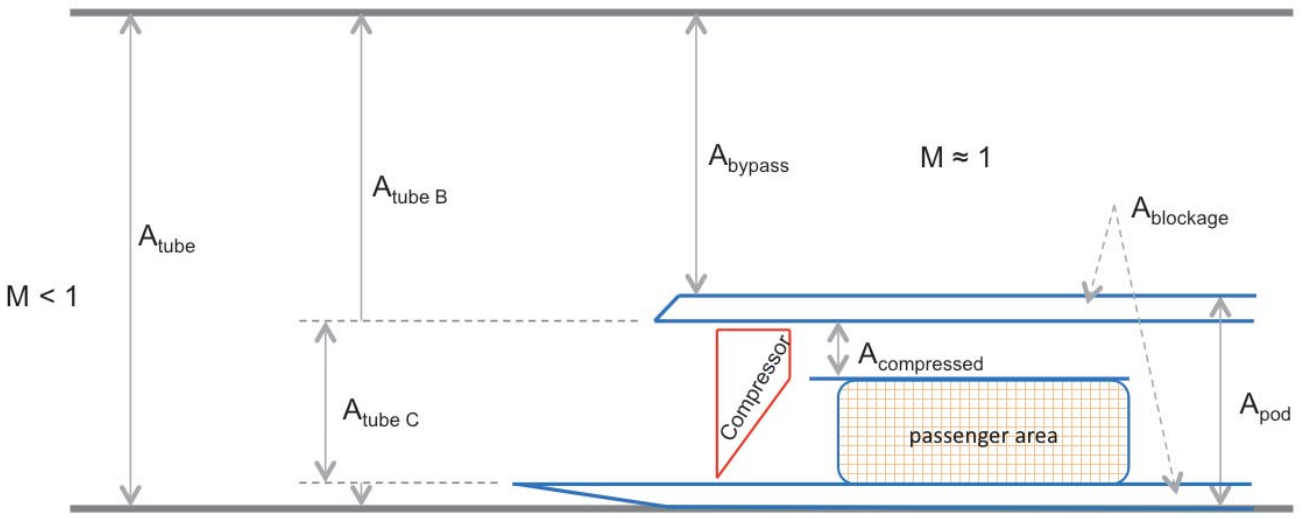

Figure 7: Longitudinal view of a hollow passenger pod that lets mass flow pass through it.

To get around this limitation, Hyperloop allows some mass flow to pass through the pod itself, thereby increasing the maximum achievable speed. This effectively splits the relative mass flow in $A_{\text {tube }}$ into two streams, $A_{\text {tube } B}$ and $A_{\text {tube } C}$, as shown in Fig. 7. If we neglect the small thickness of the walls of the passenger pod itself and assume that $A_{\text {tube } C}$ is equal to the $A_{\text {pod }}$, then it follows that $A_{\text {tube } B}$ will be equal to $A_{\text {bypass }}$. Without any area contraction the flow in the bypass will not accelerate, staying equal to $M_{\text {pod }}$. The challenge is that the pod cannot be perfectly hollow. It must provide some space for the passengers to sit, causing blockage in the flow. This problem can be handled by adding a compression system to the pod that will force the air into a smaller area $A_{\text {compressed }}$, to move around the passengers. This would seem to solve all of the challenges associated with high travel speeds inside a tube discussed above and decouple $\frac{A_{\text {bypass }}}{A_{\text {tube }}}$ and $M_{\text {pod }}$.

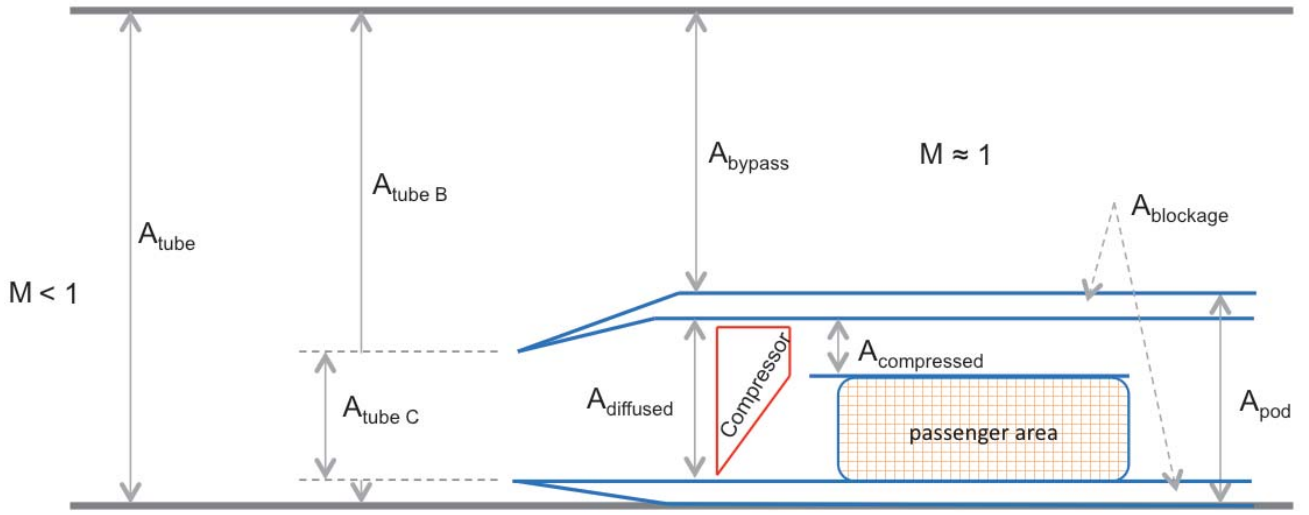

Figure 8: Longitudinal view of a hollow passenger pod with a necessary diffuser to restrict the Mach number of the flow into the compressor.

However, in order to achieve reasonable efficiency from the compression system, the Mach number of the flow at the compressor face must be limited to less than about 0.65. For $M_{\text {pod }}$ greater than 0.65, a diffuser must be added to the Hyperloop which will slow the air down before it enters the compressor. As depicted in 
Fig. 8, diffusing the flow requires expanding the area; $A_{\text {diffused }}$ must be greater than $A_{t u b e, c}$. Unfortunately this means that $A_{\text {bypass }}$ must be smaller than $A_{\text {tube } B}$, and some acceleration of the flow around the pod will occur. Once again, $\frac{A_{\text {bypass }}}{A_{\text {tube }}}$ becomes coupled to $M_{\text {pod }}$, although thanks to the compression system this is now much less restrictive than if the pod is sealed.

Figure 9 shows the rise in achievable pod Mach number based on increasing amount of hollowness. This increase is dependent on the amount of space taken up by the pod outer structure, which is a ratio of dimensions shown in Fig. 8 as a non-dimensional "blockage factor" equal to $\frac{A_{\text {diffused }}}{A_{\text {pod }}}$. The higher the factor, the lower the blockage from the pod. For a nominal tube diameter of 4 meters, the maximum pod Mach increases from 0.66 for a closed pod (not shown) to 0.82 for a hollow pod with a blockage factor of 0.9. To a lesser extent, the max speed is also dependent on the compressor entrance Mach number. Changing the allowable compressor entrance Mach number by \pm 0.05 changes the maximum pod MN by roughly \pm 0.01 . Modifying the blockage factor by \pm 0.05 changes the maximum pod MN by roughly \pm 0.03 . It should be noted that all of these trends are unaffected by the tube pressure, however, static pressure affects the compressor flow and power requirements discussed next.

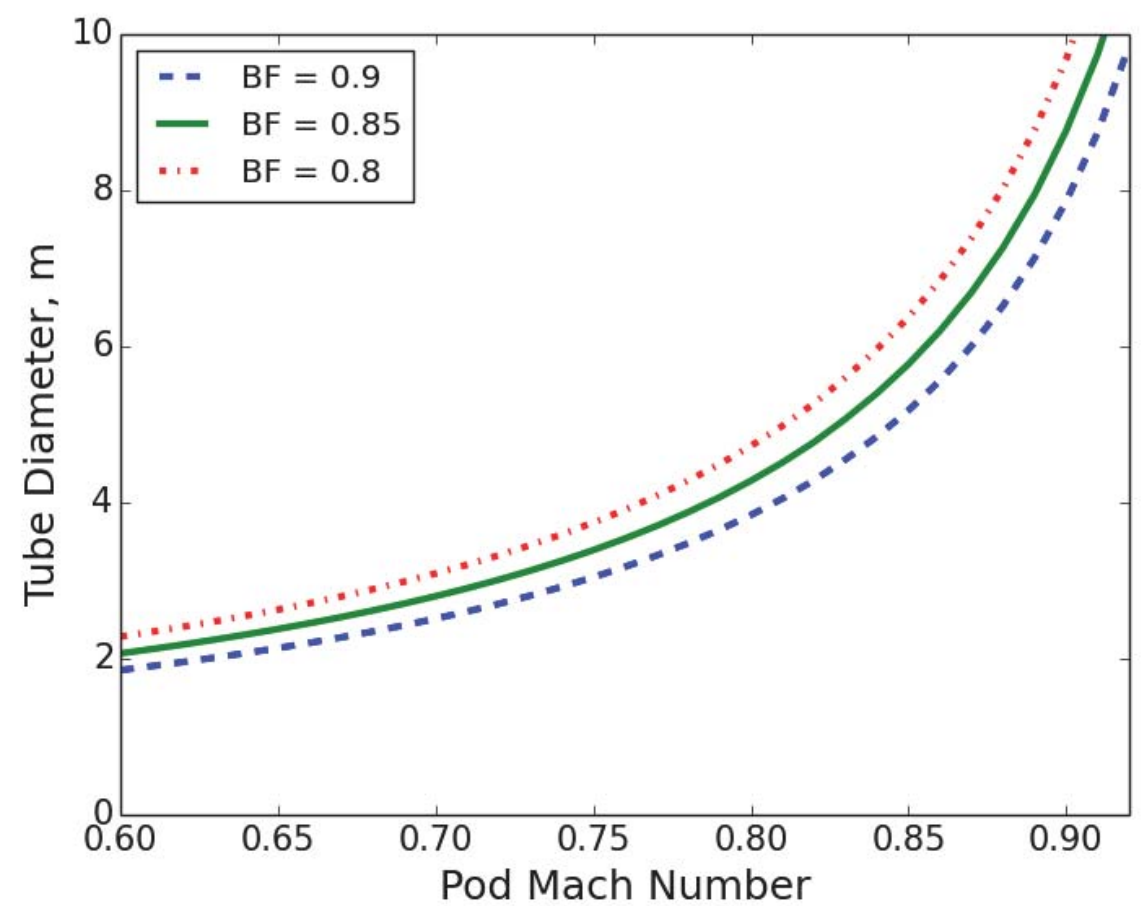

Figure 9: Exponential relationship between pod speed and required tube diameter, for three blockage factors $\frac{A_{\text {diffused }}}{A_{\text {pod }}}$.

\section{Compressor Power and Battery Sizing}

The on-board compression system serves three purposes. It provides a means of increasing the maximum pod speed over a closed pod, supplies pressurized air to the air bearing system, and provides a small amount of thrust. The second function requires a minimum airflow to provide the fluid pressure necessary to support the vehicle weight. A thermodynamic analysis of the compressor system is also necessary to estimate onboard power requirements and overall heat rise of each pod. The compression cycle is comprised of an inlet, two compressors, a nozzle, and multiple ducts leading to air bearings. The design deviates from the original proposal by removing two heat exchangers for reasons explained in section V. The system, shown previously in Fig. 4, is modeled as a one-dimensional cycle, representing components as thermodynamic processes that are subsequently chained together. Each component is responsible for calculating gas properties across its boundaries and appropriately enforcing conservation equations across the entire system.

This tool, later named "PyCycle", was built within the openMDAO framework and leverages Cantera ${ }^{5}$ for 
thermodynamic calculations. The model predicts the instantaneous power consumption of the compressors, temperature and pressure rises, and upstream conditions necessary to supply sufficient airflow to the bearings. These power requirements are a function of the chosen geometry, creating the feedback loop B in Fig. 2. Combined with the mission profile described below, these requirements impact battery sizing.

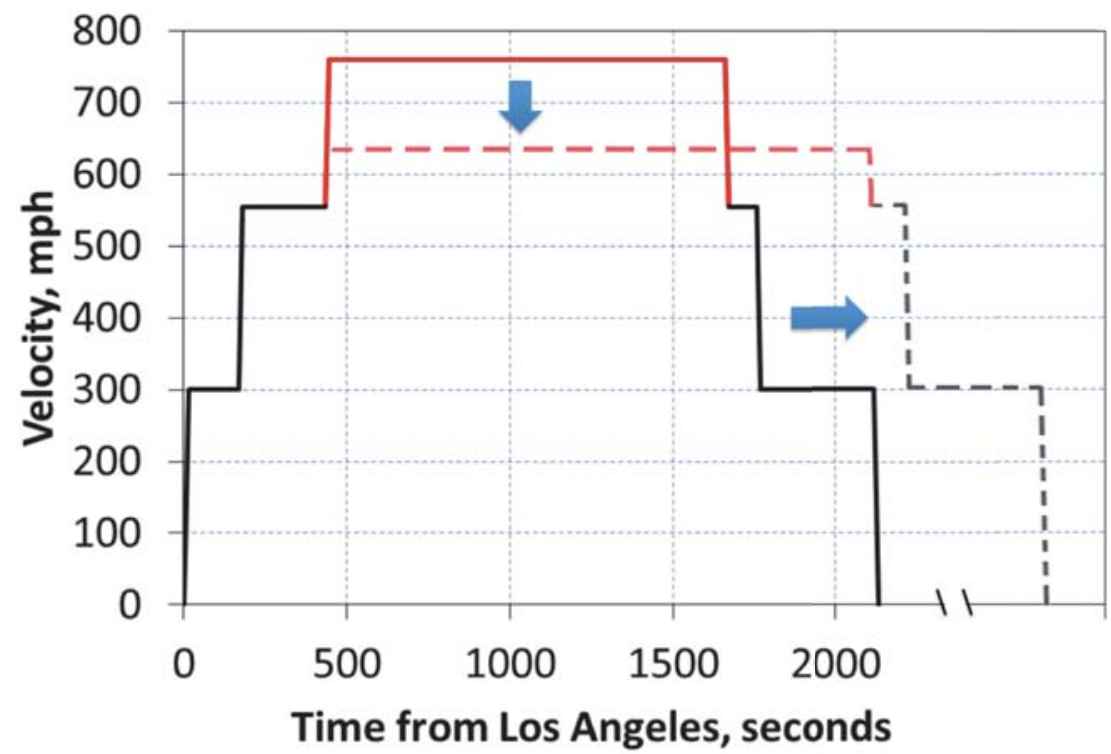

Figure 10: Velocity profile described in the original proposal, with the programmatically modified section highlighted in red.

The notional velocity profile described in the original proposal, shown by the solid line in Fig. 10, is programmatically modified based on the recomputed maximum velocity of the pod. The starting and final velocities of the mission profile are dictated by maximum allowable acceleration loads, while the highest plateau is governed by the choked flow limit. Therefore, the fastest portion of the trip, illustrated in red, is modified as the simulation runs and re-computes pod speed. Reducing the maximum speed also increases the total mission time, pushing the final mission segment out further as shown by the dashed lines. The integrated area under the curve represents the total length of the tube, which remains constant as the max speed varies. The speed constraints outlined earlier increase total mission time on the order of five minutes, however this will ultimately depend on the chosen tube diameter. Despite the increased mission time, the slower speeds would also allow for more forgiving acceleration loads around turns. Multiplying the calculated mission time by the maximum instantaneous compressor power consumption (plus a $30 \%$ safety margin) results in an overall battery storage requirement.

The necessary on-board battery energy was found to be negatively correlated to the max pod speed, as shown by the green curve in Fig. 11. The maximum instantaneous compressor requirements and total mission time are also directly overlaid in red and blue respectively. The negative slope of the green battery curve indicates that total mission time falls faster than the compressor power requirements increase. Although traveling at higher speeds draws more energy, the system is operated for a shorter period of time. At higher travel speeds, the total battery requirements start to flatten and the trend starts to reverse. The units are scaled such that the slopes of each curve can be more easily related to each other spatially. The overall reduction in battery size is on the order of $7 \%$, if the speed is increased from Mach 0.7 to Mach 0.9, however the analysis only considers the on-board energy requirements. It does not consider the increased power necessary for the linear accelerators to drive the pod to higher speeds. Furthermore, the battery trend is highly dependent on the velocity profile and reducing time spent at max speed could result in a positive correlation between battery size and pod speed. Additional route analyses can be found in work done by Mathworks. ${ }^{6}$ These estimated energy requirements are in agreement with Musk's work, and equate to roughly 4 battery packs from a Tesla Model-S. Notably, these estimates do not include considerations for battery cooling requirements or auxiliary power, and are based on the assertion that the compression cycle does not need to be cooled. The system-level thermal interactions and heat exchangers are analyzed next. 


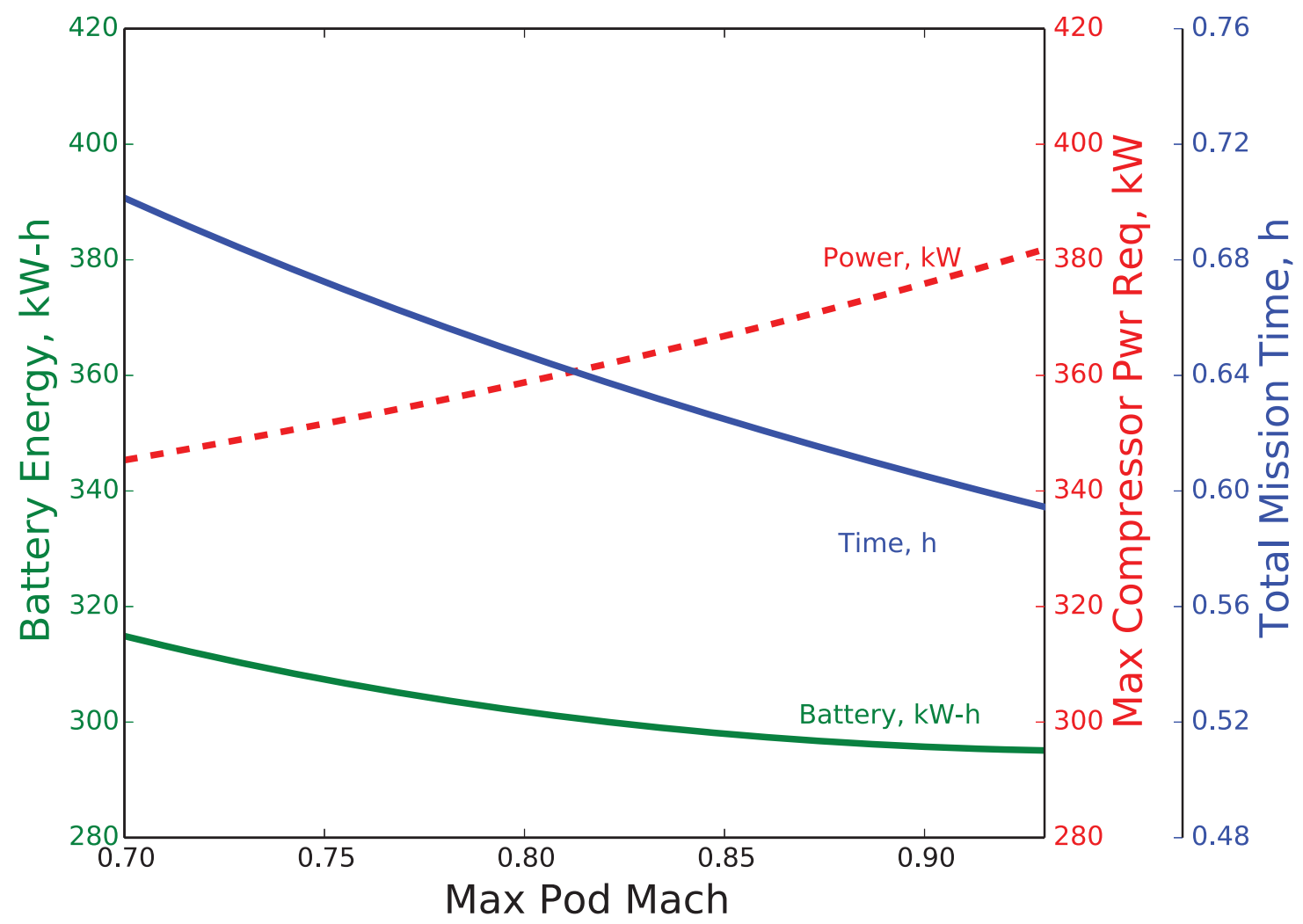

Figure 11: Battery requirements, compressor power, and mission time as a function of pod speed.

\section{Heat Exchanger and Tube Thermal Analysis}

As each pod passes through the tube, it adds energy to the air in the form of heat. Following the proposed frequency of launching a pod every six minutes, the continuous operating cycle could potentially heat the overall tube to excessive temperatures. To combat this effect, the original proposal recommends a heat exchanger that would be integrated into the compression system. These intercoolers would use water stored in on-board tanks to cool the air and assist secondary compression. The resulting steam could then be stored in a tank and offloaded once the pod reached its destination. However, initial calculations show that using water for cooling is not an ideal design for two reasons:

1) The flow rate of water needed to remove the heat added by the compressors is very large, and the sheer volume constraints of storing the resulting steam would outweigh the benefits.

2) The heat addition from each pod compressor cycle is fairly low relative to other heat transfer mechanisms occurring between the Hyperloop tube and the external environment. Even without an active on-board cooling solution, the tube temperature would be dominated by other factors.

The following two sections provide additional details about the engineering models used to draw these conclusions.

\section{Pod Cooling Requirements}

The limits and requirements of a hypothetical on-board heat exchanger can be estimated with a straightforward energy balance. The effectiveness of a heat exchanger can be described as the ratio of actual heat transfer over the maximum possible heat transfer.

$$
Q_{\text {released }}=\text { effectiveness } \cdot \underbrace{\left(T_{\text {hot }, \text { in }}-T_{\text {cold }, \text { in }}\right)\left[\dot{m} C_{p}\right]_{\text {fluid }}}_{Q_{\max }}
$$

where $Q_{\max }$ is determined by the fluid with the lowest $\dot{m} C_{p}$ product, which dictates the maximum possible heat transfer. In order to satisfy the energy balance $Q_{\text {released }}=Q_{\text {absorbed }}$ the following must be 
true,

$$
\underbrace{\dot{m}_{\text {air }} C_{p, \text { air }}\left(T_{\text {out }, \text { air }}-T_{\text {in,air }}\right)}_{Q_{\text {released }}}=\underbrace{\dot{m}_{\text {water }} C_{p, \text { water }}\left(T_{\text {out }, \text { water }}-T_{\text {in }, \text { water }}\right)}_{Q_{\text {absorbed }}}
$$

where the $T_{\text {out }}$ of each fluid is unknown. With assumed mass-flow rates and initial temperatures, a valid combination of $T_{\text {out }}$ 's of each fluid can be found through solver iteration. Valid effectiveness levels for heat exchangers can be estimated based on the Effectiveness - Number of Transfer Units (NTU) method. The effectiveness for a counter flow heat exchanger with a $\frac{C_{p, \min }}{C_{p, \max }}$ of 0.25 was chosen with air and water as the working fluids. The following conditions satisfied an energy balance with an extremely optimistically assumed effectiveness of 0.9765 , and the proposed requirement to fully cool the air back down to inlet temperatures.

Table 3: Heat Exchanger Fluid Properties

\begin{tabular}{lcccccc}
\hline Fluid & $C_{p}\left(\frac{k J}{k g-K}\right)$ & $T_{\text {in }}(\mathrm{K})$ & $T_{\text {out }}(\mathrm{K})$ & $\dot{m}\left(\frac{\mathrm{kg}}{\mathrm{s}}\right)$ & $\mathrm{Q}\left(\frac{\mathrm{kJ}}{\mathrm{s}}\right)$ & $Q_{\max }$ \\
\hline Air & 1.006 & 791 & 300 & 0.49 & -242 & 247.9 \\
Water & 4.186 & 288.15 & 416.6 & 0.45 & 242 & 247.9 \\
\hline
\end{tabular}

With a 35 minute trip, $0.45 \frac{\mathrm{kg}}{\mathrm{s}} \cdot 60 \frac{\mathrm{s}}{\mathrm{min}} \cdot 35 \mathrm{~min}=945 \mathrm{~kg}$ of standard temperature/pressure water would need to be carried. Beyond weight concerns, the density of saturated steam at the given temperatures is on the order of $1-2 \frac{\mathrm{kg}}{\mathrm{m}^{3}}$ meaning that the resulting volume necessary for 2-4 atm steam tanks would be impractical given a cross-section smaller than $8 \mathrm{~m}^{2}$. Depending on the tank temperature and pressure conditions, these tanks could exceed a hundred meters in length. This doesn't even account for the second stage heat exchanger, making the system nearly infeasible with water and unpressurized tanks. Various systems involving partial cooling, alternate coolants (such as liquid air), or pressurized tanks could be explored.

Further discussion of heat exchanger sizing can be found in the heat exchanger section of appendix B. The calculations explore the possibility of multi-pass heat exchangers and the logarithmic mean temperature difference of the heat exchanger is considered. ${ }^{7,8}$

\section{Equilibrium Tube Temperature}

The onboard water cooling and vapor collection system as proposed in Ref. 1 appears infeasible for reasons described above. Nevertheless some form of pod cabin cooling would be desired. If the tube temperature is not excessive, pod occupants could be kept comfortable with a relatively simple cabin air conditioning system. Cabin heat could be rejected from the refrigerant to the tube environs via conventional heat exchangers.

A simple quasi-steady equilibrium heat balance is performed to determine the approximate tube temperature. The analysis ignores heat transfer time lags and diurnal cycles. Heat is assumed to transfer and equilibrate rapidly between the pod compressor system, the rarified atmosphere inside the tube, the tube walls, and the outside environment. Further investigation is needed to determine the validity of this assumption. What in the real world would be a complex heat transfer problem is modeled here simplistically. This assessment is intended to estimate a conservative equilibrium tube temperature at full sun during the maximum heat of the day. An analysis of this level of fidelity is hoped to be sufficient to determine if a simple cabin air conditioning system could manage pod heating.

If the heat of the rarified atmosphere inside the tube exchanges rapidly with the tube wall, then the total temperature of the air entering the compressor inlet may be related to the tube wall temperature $\left(T_{t u b e}\right)$ and the pod Mach number $(M)$ :

$$
T_{t, \text { inlet }}=T_{\text {tube }}\left[1+\frac{\gamma-1}{2} M^{2}\right]
$$

Using additional isentropic relationships, the total temperature of the air exiting the pod nozzles may be written as a function of the compressor's pressure ratio $\left(\pi_{c}\right)$ and adiabatic efficiency $\left(\eta_{c}\right)$ :

$$
T_{t, \text { exit }}=T_{t, \text { inlet }}\left[1+\frac{1}{\eta_{c}}\left[\pi_{c}^{\frac{\gamma-1}{\gamma}}-1\right]\right]
$$

The heating rate due to all of the pods operating in the tube may be estimated by

$$
Q_{\text {pods }}=\dot{m} C_{p, \text { air }}\left(T_{t, \text { exit }}-T_{t, \text { inlet }}\right) n
$$


where $n$ is the number of pods in transit, $\dot{m}$ is the airflow per compressor determined by the cycle analysis, and $C_{p, a i r}$ is the specific heat of air given by the relations in Ref. 9 .

The entire 300 mile length of the tube is assumed to be unshaded and exposed to sunlight. Only direct solar irradiance $(G)$ is considered. At noon on a clear day at ground level, $G$ may be as great as $1000 \frac{W}{m^{2}}$, although it may be substantially attenuated on cloudy days. The heating rate due to solar irradiation is estimated by

$$
Q_{\text {solar }}=\underbrace{\left(1-\rho_{r}\right) c_{\text {solar }} G}_{\text {Solar Heat per unit Area Effective Area }} \underbrace{L_{\text {to }}}_{\text {tube } D_{o, t u b e}}
$$

where $\rho_{r}$ is the total, hemispherical reflectivity of the tube's exterior surface, $L_{\text {tube }}$ is the length of the tube, and $D_{o, \text { tube }}$ its outer diameter. $c_{\text {solar }}$ is a gross adjustment factor intended to account for diffuse scattered irradiance (causing $c_{\text {solar }}$ to increase), as well as shaded regions, non-normal incidence and other geometric peculiarities (causing $c_{\text {solar }}$ to decrease). Added together, $Q_{\text {pods }}$ and $Q_{\text {solar }}$ represent the incoming heating rate on the tube. At this point an interesting observation may be made. Using representative values for each of the system parameters, it is estimated that the heat generated by the pods in transit amounts to a small fraction of the total heating rate. Insolation appears responsible for more than $95 \%$ of tube heating. The impact of solar heating, of course, may be abated by coating or shading the tube's steel surface with a highly reflective and emissive material.

On the other side of the thermal balance, radiation and convection heat transfer from the tube are assumed to be the primary heat loss mechanisms. The radiative heat transfer is estimated by

$$
Q_{\text {rad }}=\underbrace{\epsilon \sigma\left(T_{t u b e}^{4}-T_{a m b}^{4}\right)}_{\text {Radiated Power per unit Area }} \underbrace{\pi L_{\text {tube }} D_{o, t u b e}}_{\text {Surface Area }}
$$

where $\epsilon$ is the total, hemispherical emissivity of the tube's exterior surface, $\sigma$ is the Stefan-Boltzmann constant, and $T_{a m b}$ is the ambient temperature. The heat transfer surface is assumed to be gray, and the surroundings are assumed to be isothermal and black. Convection heat transfer from the tube to the surroundings is estimated using a correlation of the Nusselt number: ${ }^{10}\left(\mathrm{Nu}\right.$, defined as $\frac{h D_{o, t u b e}}{k}$ )

$$
N u=\left(0.6+\frac{0.387 R a^{\frac{1}{6}}}{\left[1+\left(\frac{0.559}{P r}\right)^{\frac{9}{16}}\right]^{\frac{8}{27}}}\right)^{2}
$$

where $R a$ is the Rayleigh number $\left(\frac{g \beta}{v \alpha}\left[T_{t u b e}-T_{a m b}\right] D_{o, t u b e}^{3}\right)$ and $\operatorname{Pr}$ is the Prandtl number $\left(\frac{\nu}{\alpha}\right)$. The correlation is applicable for estimating free convection from long, horizontal cylinders. Properties for air $(h, k, \beta, \nu$ and $\alpha$ ) are based on the film temperature of the thermal boundary layer and are given by the regressions in Ref. 9. With the heat transfer coefficient $h$ known from the Nusselt number, the heat lost to free convection $\left(Q_{\text {conv }}\right)$ may be estimated from the total tube surface area.

$$
Q_{\text {conv }}=\underbrace{\frac{k \cdot N u}{D_{o, t u b e}}}_{h} \underbrace{\pi L_{\text {tube }} D_{o, \text { tube }}}_{\text {Area }} \underbrace{\left(T_{\text {tube }}-T_{\text {amb }}\right)}_{\Delta T}
$$

The equilibrium tube temperature is determined by varying $T_{\text {tube }}$ until the criterion $Q_{\text {pods }}+Q_{\text {solar }}=$ $Q_{\text {rad }}+Q_{\text {conv }}$ is satisfied. Estimating a single deterministic tube temperature, however, is insufficient. The parameters of the problem have a strong influence over the resulting equilibrium temperature. For example, ambient temperature will vary substantially. A breeze can amplify convection rates by a factor of two or three, and furthermore, it is not clear what surface properties the tube may have. For these kinds of reasons, several parameters in the problem are set to vary in a Monte Carlo probability experiment. The deterministic tube temperature model above is transformed into a stochastic model by replacing portions of its input with continuous random values. The random values are derived from probability distributions around the model's nominal values as listed in Table 4 .

A histogram and a normal distribution of tube temperature based on 15,000 samples are shown in Fig. 12. The mean tube temperature is $108^{\circ} \mathrm{F}$ with a standard deviation of $11^{\circ} \mathrm{F}$. Exceeding $137^{\circ} \mathrm{F}$ only occurs in $0.5 \%$ of the possible scenarios generated. This Monte Carlo assumes a fixed tube diameter of 4 meters, for reasons outlined in Section III. Repeating the experiment closer to the original 2 meter diameter results in a distribution with approximately the same mean and a lower standard deviation of $9.7^{\circ} \mathrm{F}$. 
Table 4: Uncertainty variables used in the Monte Carlo experiment.

\begin{tabular}{lccccc}
\hline Variable & Mode & Model & Min & Max & Std. Dev \\
\hline \hline$T_{a m b}$ & $305 \mathrm{~K}$ & Normal & - & - & $4.5 \mathrm{~K}$ \\
$G$ & $1000 \frac{W}{m^{2}}$ & Triangular & $200 \frac{W}{m^{2}}$ & $1000 \frac{W}{m^{2}}$ & - \\
$\rho$ & 0.5 & Triangular & 0.4 & 0.9 & - \\
$\epsilon$ & 0.5 & Triangular & 0.4 & 0.9 & - \\
$\eta_{c}$ & 0.69 & Triangular & 0.6 & 0.8 & - \\
$n$ & 34 & Triangular & - & - & 2 \\
$c_{\text {solar }}$ & 0.7 & Triangular & 0.5 & 1.0 & - \\
$c_{\text {conv }}$ & 1.0 & Triangular & 0.9 & 3.0 & - \\
\hline
\end{tabular}

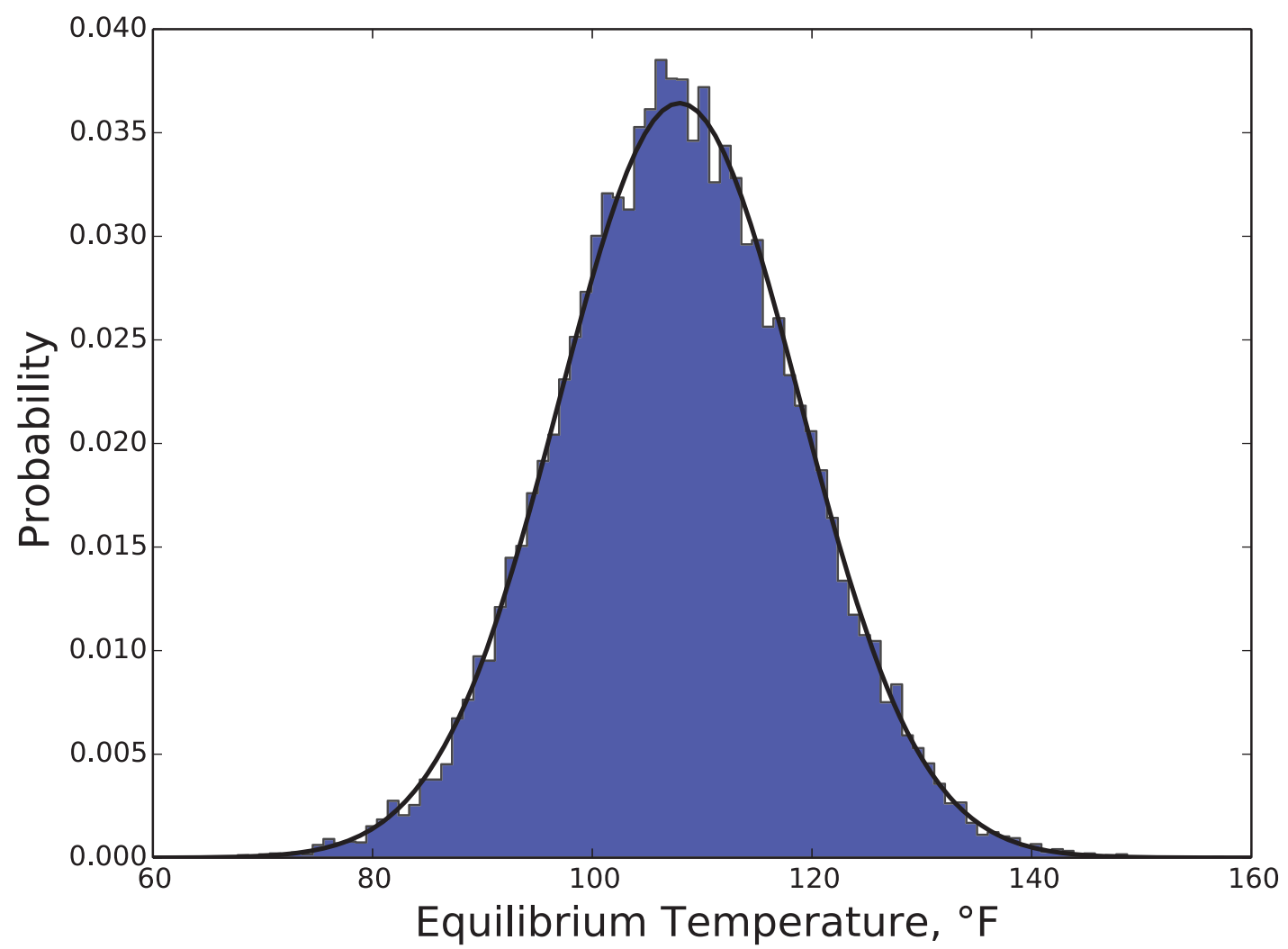

Figure 12: Monte Carlo uncertainty analysis of the equilibrium tube temperature. Histograms and normal distribution generated from 15,000 samples using a bin span of $1^{\circ} \mathrm{F}$. 
The sub-iteration of the thermal discipline then feeds back into the overall Hyperloop convergence loop, both affected by and contributing to the compression cycle, creating feedback $\mathrm{C}$ in Fig. 2. The analysis suggests that the dominant heating factors are a direct result of the massive tube structure, with only $5 \%$ of the heat contributed from the pods. The thermal management should therefore be incorporated into the tube design, rather than integrated into the volume and weight constrained pod design. Considerations for solar panel shielding as outlined in the original proposal would produce even more thermally optimistic results.

\section{Conclusions}

Using openMDAO and pyCycle, a publicly extensible model of the Hyperloop concept was created to investigate the features and assertions of the original design. The refined analysis illuminates several interdisciplinary couplings that alter two major aspects of the initial concept. First, the pod travel speed and the tube cross sectional area are linked, forcing the tube size to be to be roughly twice the diameter of the original specification, in order for the pod to reach Mach 0.8. Second, the steady-state tube temperature is dominated by ambient thermal interactions unrelated to the heat generated by the pod compression system. As a result, the two water-based heat exchangers originally prescribed for thermal management are eliminated. Although this work identifies some necessary refinements to the Hyperloop design, the core concept remains potentially feasible and warrants continued investigation. This work emphasizes the importance of maintaining a systems-level approach and provides toolsets capable of managing the growing complexity. The modeling platform is intended to serve as an easily accessible baseline that is straightforward to expand and delve deeper into this unique multidisciplinary endeavor.

\section{References}

\footnotetext{
${ }^{1}$ Musk, E., "Hyperloop Alpha," Website, August 2013. Retrieved from, <http://www.spacex.com/sites/spacex/ files/hyperloop_alpha.pdf>

${ }^{2}$ Oster, D., Kumada, M., and Zhang, Y., "Evacuated tube transport technologies (ET3): a maximum value global transportation network for passengers and cargo," Journal of Modern Transportation, Vol. 19, No. 1, 2011, pp. $42-50$.

${ }^{3}$ Gray, J., Moore, K. T., Hearn, T. A., and Naylor, B. A., "Standard Platform for Benchmarking Multidisciplinary Design Analysis and Optimization Architectures," AIAA Journal, Vol. 51, No. 10, Oct 2013, pp. 2380-2394.

${ }^{4}$ Claus, R. W., Evans, A. L., Lytle, J. K., and Nichols, L. D., "Numerical Propulsion System Simulation," Computing Systems in Engineering, Vol. 2, No. 4, 1991. doi:10.1016/0956-0521(91)90003-N

${ }^{5}$ Goodwin, D., Malaya, N., Moffat, H., and Speth, R., "Cantera: An object-oriented software toolkit for chemical kinetics, thermodynamics, and transport processes," Caltech, Pasadena, 2009.

${ }^{6}$ Rouleau, G. and Brauer, M., "Hyperloop: Not so fast!" Website, November 2013.

${ }^{7}$ Cengal, Y., Turner, R., and Cimbala, J., Fundamentals of thermal-fluid sciences, McGraw-Hill Companies, 2008.

${ }^{8}$ Turns, S., Thermal-fluid sciences: An integrated approach, Cambridge University Press, 2006.

${ }^{9}$ Clausing, A.M., "Simple Functional Representations for the Thermophysical Properties of Gases at Standard Pressure," ASHRAE Transactions, Vol. 88, Part 2, 1982, pp. 645-654.

${ }^{10}$ Churchill, S.W., and Chu, H.H.S., "Correlating Equations for Laminar and Turbulent Free Convection from a Horizontal Cylinder," Int. J. Heat Mass Transfer, 18,1049, 1975.
} 


\section{A. Sample Source Code and External Contributions}

\section{Github}

The presented models were built exclusively using freely-available, open-source tools. The code requires OpenMDAO, a framework serving as the backbone for subsystem integration, optimization and workflow management. As the Hyperloop design evolves, an ever increasing number of design variables will need to be managed and optimized. OpenMDAO can provide the means to manage this rapidly growing complexity in an efficient and flexible manner that will allow the model to grow as the necessary refinements are made. External contribution is greatly encouraged to expand, refine or replace models with higher fidelity analyses.

The entire source code for both the analysis and the generated figures can be found on github at: <https://github.com/OpenMDAO-Plugins/Hyperloop>

Online documentation can be found at: <http://openmdao-plugins.github.io/Hyperloop> This plugin is designed to be a jumping off point for community contributions to help crowd source the development of the Hyperloop concept. The readme on the github repository walks through basic installation steps, further support can be found through the main OpenMDAO docs. The basic structure of an assembly is explained in the usage section of these docs.

\section{Usage Example}

To use the Hyperloop model, run the file src \hyperloop \hyperloop_sim.py in the Hyperloop repository. Each assembly is structured similarly and the following walk-through shows the basic layout for setting up a model.

The file starts out with some library imports and the Input/Output variable definition of the HyperloopPod assembly.

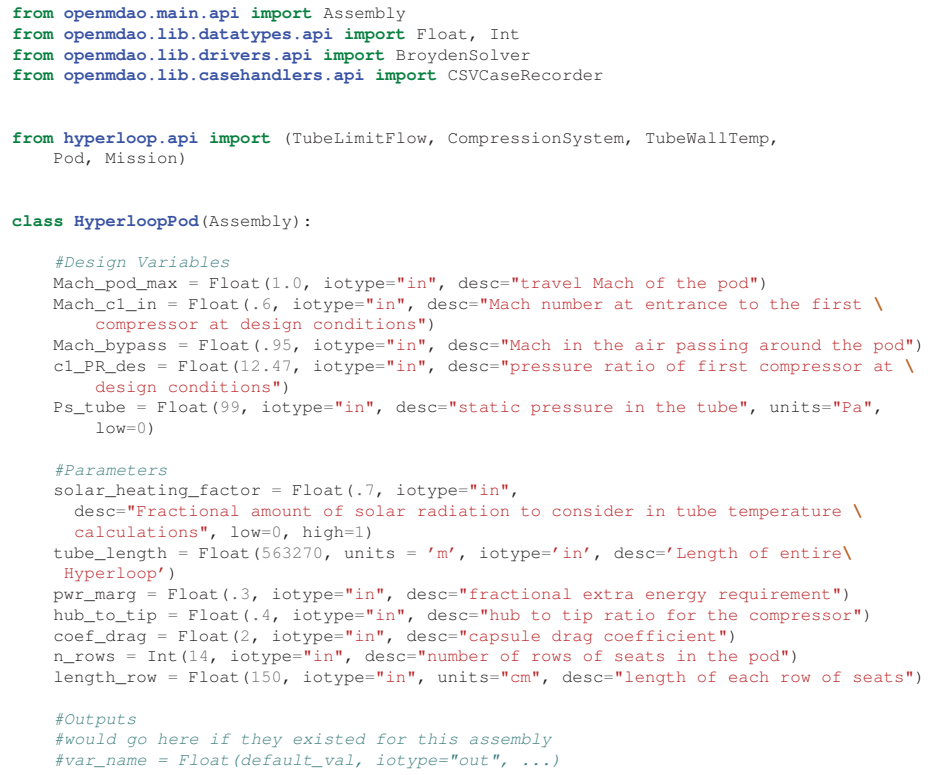

Next is the configure method, which is used to wire up the assembly components like the diagrams shown in the model layout section.

First add an instance of each component class, then connect variables to and from each component.

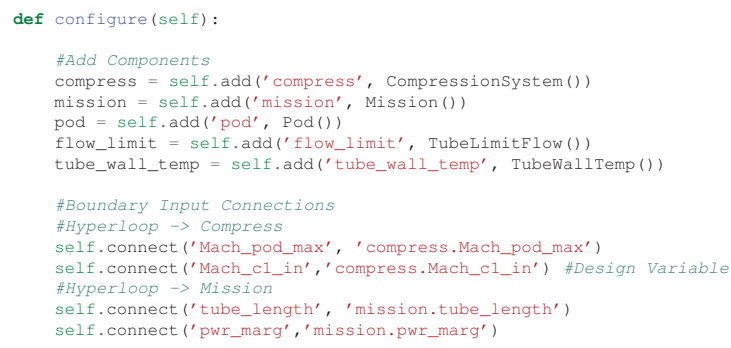




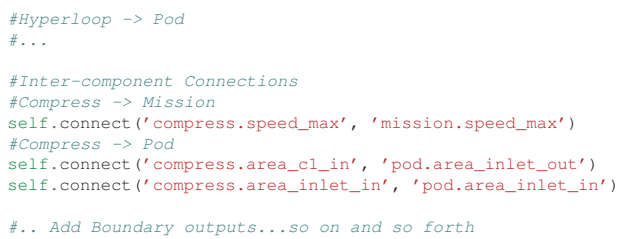

Since assemblies often require iteration and convergence, a solver is then added. Each added parameter gives the solver variables to vary, until all declared constraints are satisfied.

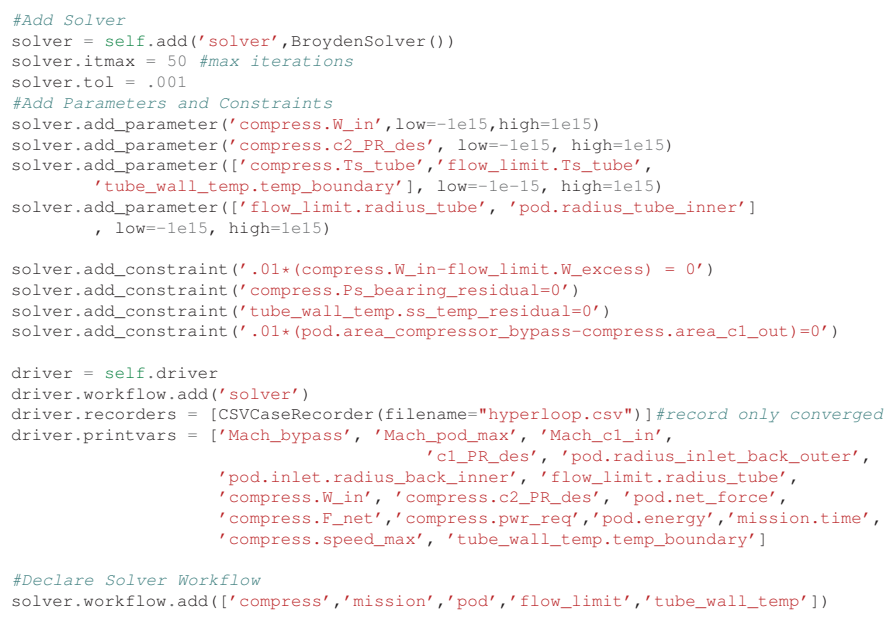

The final if _name__==_-_main__: section works the same as any other python script. This trick allows the user to set up conditional inputs and parameters for the file to run by itself, rather than in conjunction with the rest of the optimization. Running stand-alone is much more convenient when initially building a component and debugging.

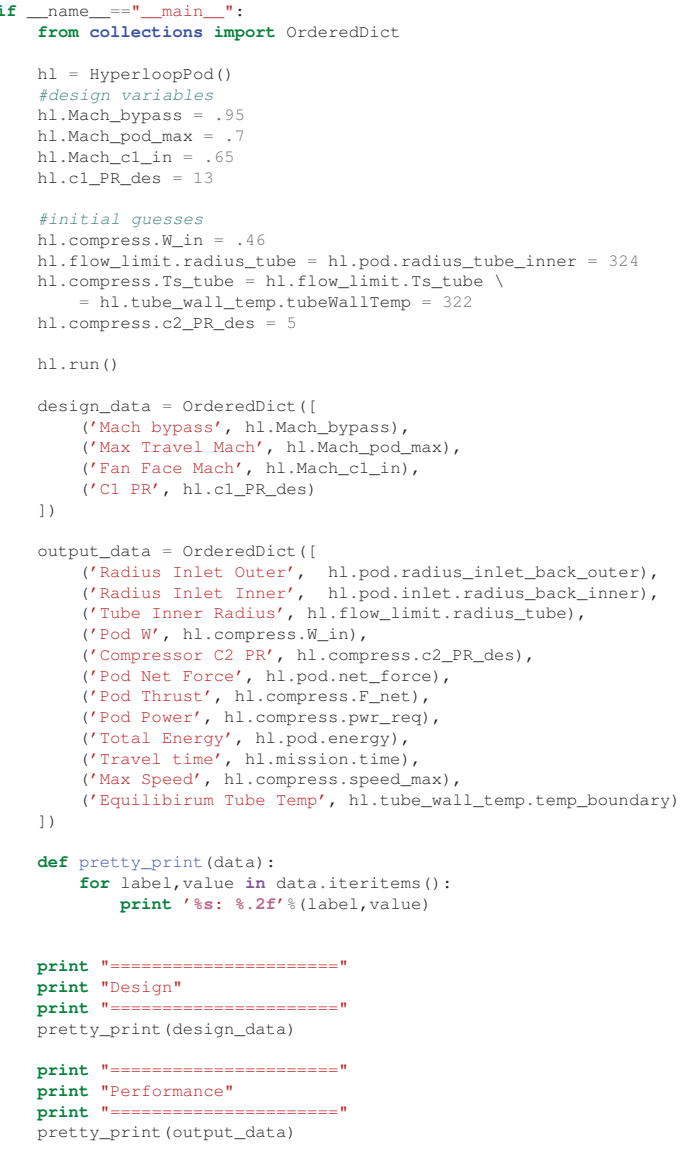




\section{B. Subsystem Modeling Theory and Potential Future Road Map}

The current model of the Hyperloop focuses on some of the primary sub-systems that operate within the pod. However, there is much more analysis that needs to be done to build a complete Hyperloop model. For the sake of conciseness, each section serves as a general summary. The reader is recommended to refer to the actual source code and included documentation for full implementation details. The current model omits economic, structural, safety, and infrastructure considerations; these areas become more prominent once the core engineering concept is deemed sufficiently feasible. These aspects are equally important to the overall design and they represent the next required step in producing a viable Hyperloop design concept. Below, is a brief summary in which various disciplines are suggested as logical next steps for the engineering aspects of the analysis.
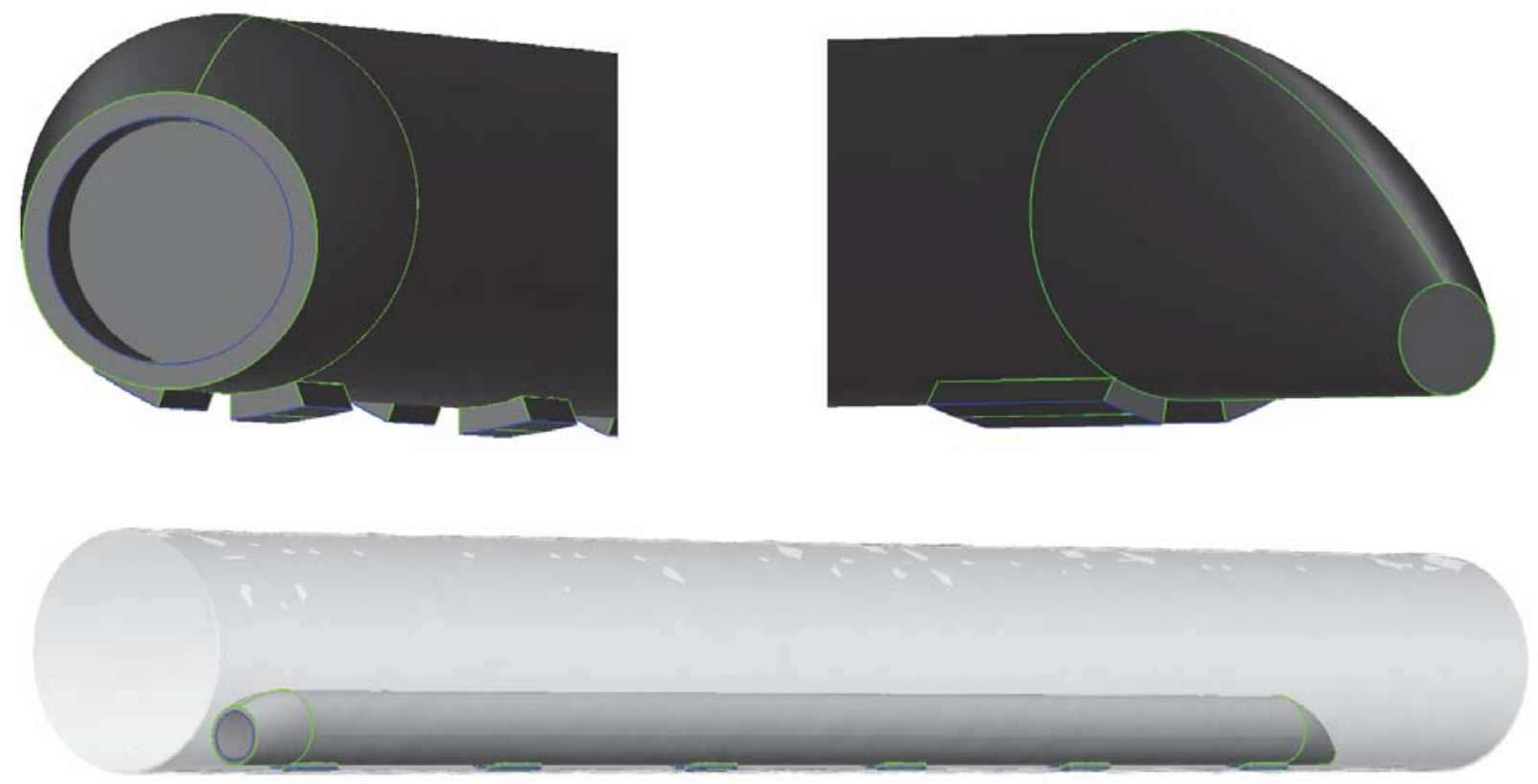

Figure 13: Hyperloop conceptual rendering. Calculated dimensions for an inlet (left), nozzle (right), and full assembly (bottom) for a pod speed of Mach 0.8. Geometry rendered in OpenCSM, a parametric solid modeling tool.

\section{System Design Optimization}

The current baseline appears to be a feasible design, but the design space is large (and will grow with additional models) and needs to be more fully explored. Overall, the goal of the Hyperloop design should be to find the right compromise between maximum passenger throughput, minimum travel time, and minimum cost per trip. The following are some major open questions about the Hyperloop design space:

1) What is the relationship between overall energy usage and tube pressure? Would a slightly higher pressure lower the overall energy consumption by reducing vacuum pump effort more than it increases power requirements for the pod?

2) What is the best combination of pressure ratios for the compression system? Does the bypass air need to be pressurized so highly?

3) What is the best size for the tube diameter? Larger diameters will increase pump effort, but decrease pod power usage; what is the best balance? Could a larger diameter coupled with a slightly higher pressure provide superior performance? 


\section{Geometry}

This model makes some simple geometric calculations, however a real parametric geometry model needs to be included in the optimization. This model is necessary to properly consider the layout and packaging issues involved in the design. It also needed to do more complete structural analyses on the pressurized containers, as well as to do an aerodynamic shape optimization of the outer shape.

Some alternate configurations could possibly considered as well. Although the length of the capsule would grow by a factor of 2 , it might be possible to put the seats in a single file layout to reduce the overall tube dimensions. The effect of this change on the overall system is not obvious and needs to be studied.

Following the other analyses used thus far, the geometry model needs to be built in an freely available, open-source geometry system so that it can be freely shared with the rest of the model.

\section{Battery and Motors}

The initial estimates of battery size and weight rely on elementary calculations. As noted, the power requirements amount to roughly 3 to 5 battery packs from a Tesla Model-S. Much better weight and size estimates for these off-the-shelf batteries need to be integrated.

No work has been done to size the motors or account for any cooling requirements they might need. Although the current results indicate that a cooling system for the compressed air is not needed, it may still be necessary to cool the batteries and motors. The power requirements, weight, and space needs of such cooling systems needs to be considered.

\section{Air Bearings}

The current models assume a fixed mass flow requirement for the air bearing system. A more accurate model would account for the overall weight of the pod, the pressure of the air, and the overall bearing size. A more detailed bearing model should be coupled to the compression system model to ensure a feasible design is achieved.

In addition, some investigations need to be made into the lower speed operation of the pod. It's possible that splitting the compression system into two independent paths would be beneficial if the bearings require a relatively constant mass flow rate and pressure. This would allow a more flexible operation of what is currently the first stage compressor. The original proposal also mentioned the use of aircraft landing gear at substantially lower speeds.

\section{Vacuum Pumps}

The current model indicates that a tube with around a 4 meter diameter will be needed to reach the high velocities to keep the travel time to around 35 minutes. The size of the tubes will impact the key power requirements for the vacuum pumps. The size will impact the peak power requirements to pump the tubes

down in a reasonable time, and the steady state power requirements to maintain the high vacuum in the tube. Both of these aspects need to be modeled and incorporated into the system models.

\section{Solar Power Generation}

One of the proposed features of the Hyperloop concept is its near net-zero energy consumption, via the inclusion of solar panels along the length of the tubes. Models are needed to predict, based on geographical location, weather, and time of year, how much power could be produced on an ongoing basis from such a solar panel system.

The power production and power consumption of the Hyperloop system need to be compared. Even assuming you can reach a net zero energy usage on an average basis, the timing of the production and consumption has a strong impact on how much energy storage is necessary in the overall system. This will have an impact on its overall cost.

\section{Pod Structural Design}

The passenger pod is, from a structural perspective, a pressure vessel experiencing a fairly high pressure ratio of around 1000. The original design concept calls for a non-circular pressure vessel which raises some 
structural design issues. It's possible to design an effective structure using modern aircraft grade composites technologies, but it's possible that a round cross section could allow for a more traditional construction and reduce costs. Structural models considering composites and metallic construction are needed.

\section{Component Mass Estimation}

The current model does not include any significant weight estimation. Every part of the model needs to have weight estimates added.

\section{Linear Accelerators}

These are not considered at all currently. However, they will obviously need to be modeled as part of the mission analysis work.

\section{Route Optimization}

The current mission analysis takes the velocity profile in the original proposal as a given. A more advanced analysis needs to be constructed which accounts for actual passenger G-load constraints based on an optimal route and speed profile given existing California infrastructure .

\section{Heat Exchanger Design}

In spite of the results in the capsule cooling section, on-board cooling could possibly be used to partially fulfill cooling requirements. As a basic exercise a hypothetical baseline heat exchanger model was developed to investigate the weight and sizing requirements of an on-board water cooling system using the Logarithmic Mean Temperature Difference (LMTD) method. ${ }^{78}$ The exchanger was sized to remove all excess heat generated by the two compressors using a pedagogical shell and tube design. Based on the temperature restraints and exhaust flow rate determined by the cycle model, necessary water flow rates were calculated to ensure an energy balance. Given a predefined heat exchanger cross-section, fluid flow regimes and heat transfer coefficients were obtained. The combination of all of these elements provide a first-cut approximation of tank sizes, total heat exchanger volume, and pumping requirements.

Given:

-For simplicity, only a single heat exchanger is designed (to cool down the air coming off the first compressor stage)

-Sized as a classic shell and tube heat exchanger

-Input and output temperatures are known for each fluid

-Temperature change across the heat exchanger cannot be so large that $C_{p}$ changes significantly

-Rigorously defined for double-pipe(or tubular) heat exchanger

With a chosen cross-sectional area of pipe and annulus, and known $Q$ and $\dot{m}$, the velocity of each fluid can be determined.

$$
\dot{m}=\rho A V \ldots \text { therfore } \ldots V=\frac{Q}{\rho A C_{p}\left(T_{\text {out }}-T_{\text {in }}\right)}
$$

The hydraulic diameter (characteristic length) of a tube can also be calculated as,

$$
\begin{aligned}
& D_{h}=\frac{4 A_{f}}{P_{f}}=\frac{4 \pi\left(I D_{a}^{2}-O D_{p}^{2}\right)}{4 \pi\left(I D_{a}+O D_{p}\right)}=I D_{a}-O D_{p} \\
& D_{\varepsilon}=\frac{4 A_{f}}{P_{h t}}=\frac{4 \pi\left(I D_{a}^{2}-O D_{p}^{2}\right)}{4 \pi\left(I D_{a} * O D_{p}\right)}=\frac{I D_{a}^{2}-O D_{p}^{2}}{O D_{p}}
\end{aligned}
$$

Based on the geometry, kinematic viscosity $v$, dynamic viscosity $\mu$, thermal conductivity $k$, and velocity of the fluids the following non-dimension values can be calculated

Reynolds Number: (inertial forces/ viscous forces) $R e=\frac{V D_{h}}{v}$

Prandtl Number: (viscous diffusion rate/ thermal diffusion rate) $\operatorname{Pr}=\frac{C_{p} \mu}{k}$

Based on the flow regimes determined above, the Nusselt Number.. can be calculated. The Dittus-Boelter equation is used in this case, 
Nusselt Number: (convective heat transfer / conductive heat transfer) $N u=0.023 *\left(\operatorname{Re}^{4 / 5}\right) *\left(\operatorname{Pr}^{n}\right)$ where $\mathrm{n}=0.4$ if the fluid is heated, $\mathrm{n}=0.3$ if the fluid is cooled.

Subsequently the convective heat transfer coefficient of each fluid can be determined, $h=\frac{N u * k}{D_{\varepsilon}}$

All of these terms can then be used to calculate the overall heat transfer coefficient of the system,

$$
\begin{gathered}
U_{o}=\frac{1}{\left(\frac{A_{o}}{A_{i} h i}\right)+\left(\frac{A_{o} \ln \left(\frac{r_{o}}{r_{i}}\right)}{2 \pi k L}\right)+\frac{1}{h_{o}}} \\
\Delta T_{L M}=\frac{\Delta T_{2}-\Delta T_{1}}{\ln \left(\frac{\Delta T_{2}}{\Delta T_{1}}\right)} \\
\Delta T_{1}=T_{\text {hot }, \text { in }}-T_{\text {cold }, \text { out }} \\
\Delta T_{2}=T_{\text {hot }, \text { out }}-T_{\text {cold }, \text { in }}
\end{gathered}
$$

allows the length to be determined for a single pass heat exchanger.

$$
q=U_{o} \pi D_{o} L \Delta T_{L M}
$$

Further calculations for the multipass heat exchanger can be found in the source code. 